\title{
Transition to the Cloud
}

\section{A Vendor Perspective}

\author{
Hedman, Jonas; Xiao, Xiao
}

Document Version

Accepted author manuscript

\section{Published in:}

Proceedings of the 49th Hawaii International Conference on System Sciences (HICSS 2016)

DOI:

10.1109/HICSS.2016.494

Publication date:

2016

\section{License \\ Unspecified}

Citation for published version (APA):

Hedman, J., \& Xiao, X. (2016). Transition to the Cloud: A Vendor Perspective. In R. H. Sprague, \& T. X. Bui (Eds.), Proceedings of the 49th Hawaii International Conference on System Sciences (HICSS 2016) (pp. 39893998). [7427679] IEEE. Proceedings of the Annual Hawaii International Conference on System Sciences https://doi.org/10.1109/HICSS.2016.494

Link to publication in CBS Research Portal

\section{General rights}

Copyright and moral rights for the publications made accessible in the public portal are retained by the authors and/or other copyright owners and it is a condition of accessing publications that users recognise and abide by the legal requirements associated with these rights.

Take down policy

If you believe that this document breaches copyright please contact us (research.lib@cbs.dk) providing details, and we will remove access to the work immediately and investigate your claim. 


\section{Transition to the Cloud: A Vendor Perspective Jonas Hedman and Kiao Kiao}

Article in proceedings (Post print version)

This article was originally published in HICSS 2016: 49th Hawaii International Conference on System Sciences. red. / Tung Bui; Ralph Sprague. Piscataway: IEEE, २016. Pp. 3989-3999.

Uploaded to Research@CBS: Januar २०16

(ㄱ २०16 IEEE. Personal use of this material is permitted. Permission from IEEE must be obtained for all other uses, in any current or future media, including reprinting/republishing this material for advertising or promotional purposes, creating new collective works, for resale or redistribution to servers or lists, or reuse of any copyrighted component of this work in other works. 


\section{Transition to the Cloud: A Vendor Perspective}

\author{
Jonas Hedman \\ Department of IT Management, Copenhagen \\ Business School \\ jh.itm@cbs.dk
}

\author{
Xiao Xiao \\ Department of IT Management, Copenhagen \\ Business School \\ xxi.itm@cbs.dk
}

\begin{abstract}
The rising of cloud computing has dramatically changed the way software companies provide and distribute their IT product and related services over the last decades. Today, most software is bought offthe-shelf and distributed over the Internet. This transition is greatly influencing how software companies operate. In this paper, we present a case study of an ERP vendor for SMB (small and mediumsize business) in making a transition towards a cloud-based business model. Through the theoretical lens of ecosystem, we are able to analyze the evolution of the vendor and its business network as a whole, and find that the relationship between vendor and Value-added-Reseller (VAR) is greatly affected. We conclude by presenting critical issues and challenges for managing such cloud transition.
\end{abstract}

\section{Introduction}

More than just a buzzword, cloud computing as a trend is here to stay. Nowadays, it has been increasingly more widely accepted such utility-like way to deliver and consume IT recourses. Industrial reports have suggested that corporate IT spending on cloud computing will increase by $42 \%$ in 2015 , and by year 2018 , more than $60 \%$ of the companies will have at least half their software and infrastructure in cloud [9].

The National Institute of Standards and Technology (NIST) defines cloud computing as " $a$ model for enabling convenient, on-demand network access to a shared pool of configurable computing resources (for example, networks, servers, storage, applications, and services) that can be rapidly provisioned and released with minimal management effort or service-provider interaction" [27]. There are three commonly recognized delivery models of cloud computing, namely software-as-a service (SaaS) (e.g. Salesforce.com cloud-based customer relationship management software delivered over the Internet), platform-as-a-service (PaaS) (e.g. Google's App Engine upon which various application can be developed, distributed and hosted), and infrastructure-as-a-service (IaaS) (e.g. Amazon Web Service that offers remote computing services) $[19,23]$.

The underlying technology of cloud-based IT solutions is not new and has been in existence for a long time. However, the maturation of cloud computing as a new business model is deemed to be revolutionary. The advantages provided by this new way of consuming computing resources, such as low/minimal entry costs, pay-as-you-go mode, and great flexibility and scalability, have on the one hand levelled the playing field for small and medium organizations with less resources, and on the other hand provided productivity boost, cost efficiency, and innovation potential for all organizations $[23,40]$. Existing research on cloud computing, though still in its nascent stage, has investigated various issues on adoption of cloud computing from user (individual or organizational) perspective, including adoption intention [5,16,32], industry specific risk benefit analysis [26], economic and structural impact of adoption [28,37], and governance related issues associated with cloud-based solutions [43].

Despite the academic interests being focused on the user side, the profound influences brought about by cloud computing are also present on the vendor side. Over the recent years, we have seen new players, such as Salesforce.com and Amazon, introducing the new business models and obtaining first-mover advantages. In parallel, traditional software vendors, such as Microsoft and SAP, are also making the transition to cloud-based business models [11]. An industrial report back in 2012 showed that all IT vendors have already more or less engaged in cloud using diversified strategy, and though the landscape of the vendor market has being shifting towards the new delivery mode, major cloud vendors are still dominated by the incumbents who managed to make the transition with a few exceptions (e.g., Salesforce, Amazon, and Google) [36]. For instance, with a comprehensive strategy focusing on "cloud-first design," Microsoft is going full force with the transition to cloud on SaaS, PaaS and IaaS 
levels; whereas SAP managed the shift towards offering their software on cloud through buying and partnering with other vendors at other levels [8]. For these adapters, the shift towards cloud is more than just delivering IT solutions in a new way. More importantly, it also concerns with how to manage the whole value chain and business network under the new circumstances, as cloud computing symbolizes a paradigm transition regarding IT servitization [3,23].

While existing literature on cloud computing show great interest in uncovering whether and how IT users adopt the technology, the conceptual understanding of how these transition processes for IT vendors go about are still scarce. Therefore, we choose to approach the phenomenon of cloud computing transition from the software vendor side in a business-to-business (B2B) setting, a context that is still rarely studied. More specifically, we are interested in the transition of traditional make-toshelf software vendor to a cloud world and correspondent business model. Our intention is to uncover the management challenges surrounding this process caused by technological evolution, and hence shed light on not only the transition process of the organization itself, but also the evolution of the larger ecosystem that is built around the existing business model.

We use a revelatory case study by focusing on a Nordic ERP vendor and its transition towards a SaaSbased cloud model. We use the ecosystem literature as theoretical lens, since previous research has concluded that contemporary software developments cannot be viewed in isolation, and thus, one has to consider the system and infrastructure they are part of [1]. Thereby, contributing towards an increased understanding of the effects of cloud computing per se, also to business transition in general, we seek to explain how the cloud computing, as a technology innovation, affects the software vendor ecosystem.

\section{Literature Review}

In this section, we review cloud computing related literature, with a specific emphasis of the studies taking the vendor perspective.

More often than a technological innovation, cloud computing is deemed as an innovative business model of delivering IT services [23]. The current market of cloud computing has seen the emergence of new players as well as transition of established ones operating at all three levels: Amazon, EMC, and AT\&T in providing raw computing and data storage services, IBM, Google, and Microsoft in providing platform services, and finally Salesforce.com and
SAP in providing applications and enterprise systems $[23,40]$.

From the customer perspective, it has been widely discussed in the literature the unique characteristics associated with cloud-based IT solutions that differ from the traditional ones and hence provide potential benefits for organizations that have not been realized before. To begin with, cost efficiency is among the most important factor that attracts cloud adoption $[2,40]$. On one hand, the pay-as-you-go or subscription-based payment model has liberated organizations from upfront capital investment when it comes to purchasing IT solutions [25], and hence significantly lowered the entry barrier of acquiring IT resources for smaller organizations [23]. On the other hand, for larger organizations, cost efficiency can be achieved through consolidating data centers or locating data centers in places with lower operation costs [10]. Other benefits of cloud computing include increased elasticity (the capability to scale up and down IT resources) $[19,23]$, decreased demand for IT skills or expertise (as IT operations are outsourced through cloud computing technologies) [21], and enhanced innovation potential $[23,40]$. At the same time, cloud computing related concerns, such as privacy and security issues [30], and the phenomenon of shadow IT and loss of control for central IT department within organizations [43], have also been largely debated as risks associated with cloud computing adoption. Existing literature on cloud computing has focused on the user adoption by engaging in the discussions of pertinent risks and benefits for a certain organization [42] or a specific industry [26].

However, from the vendor perspective, how such new model of IT delivery is associated with new ways and mindset in doing business has not been addressed to the same extent. For established (incumbent) players who are adapting to the new market, the transition is multi-facet. First of all, the business model of cloud computing implies a different revenue stream: instead of relying on upfront license fee, IT vendors are now collecting periodic subscription fee, suggesting that the key to survival under the new circumstance is to keep the cash flow going, which, in other words, means retaining customers [20]. Financially, this renders as a challenge, as such delivery model is considered to have significantly decreased switching costs for customers [11].

Second of all, cloud computing is an instance of the latest shift towards servitization of IT or "information systems in services" [3]. When IT solutions are no longer provided as physical products but as services over the Internet, customers' 
expectations and requirements also change accordingly $[6,40]$. For instance, Venters and Whitley [40] have uncovered and classified different desires from cloud users, which suggests that cloud computing is expected to provide equivalent services as traditional solutions, and more, namely variety, abstraction, scalability from a technical point of view, and simplicity, creativity, and efficiency from a service point of view. Therefore, it can be argued that IT vendors nowadays are providers of IT capabilities, rendering profound implications for how to operationalize IT services in correspondent ways, and also how to handle customer interactions. For instance, many of the cloud vendors mentioned above operate around a user community where open innovation is flourishing [19], suggesting a paradigm shift from treating customers as value exchangers to value co-creators [39].

Additionally, IT vendors do not exist in isolation, but collaborate with a wide range of partners, e.g. Value-added-Resellers (VAR), who can be retailers, mediators, and consultants [31]. Such industrial partnership is also likely to be influenced by the new business model of cloud computing which provides the opportunities for IT vendors to sell directly to the customers and hence eliminate middle links (i.e., disintermediation). On the other hand, other types of mediators emerge: the so-called integrators or enablers who provide services to "facilitate the delivery, adoption, and use of cloud computing" [23:183]. Therefore, one could argue that the transition is the one of the whole ecosystem surrounding the IT industry, as we see old players going out of business, and new players of various types emerging to power.

\section{Ecosystem}

We adopt the lens of ecosystem in understanding the transition of IT vendors in light of cloud computing, so as to understand the change across the industrial network. The term ecosystem originally comes from biology [38] and was popularized in business research by Moore [24]. An ecosystem is defined as a co-opetitive environment in which symbiotic relationships are formed to create mutual value for its stakeholders $[4,33,34]$. In the literature, four features of ecosystems stand out.

First, an ecosystem is characterized by simultaneous competition and cooperation, so called co-opetition [7,41]. Micro-level analysis depicts some stakeholders as direct competitors, while macro-level analysis depicts the same stakeholders as mutually dependent on the success of the ecosystem as a whole. In some setting there may be some degree of collaboration with other stakeholders in the ecosystem, resulting in strategic networks.

Second, a key success factor for a prosperous ecosystem is the relationship among the stakeholders in the ecosystem. For the individual stakeholders, this is partly a matter of managing resource dependencies. Stakeholders in the ecosystem that control important resources can exercise power over other stakeholders and can capture a larger part of the ecosystem's total revenues. However, if the stakeholders that control key resources hold the "keystone advantage" are exercising too much pressure relative to the other stakeholders, it might be fatal to the ecosystem as a whole, and as a consequence, may damage the business of the keystone stakeholders as well [18].

Third, ecosystems have observable differences. For example, within a business ecosystem, certain stakeholders can have closer relations than other stakeholders. One can even talk about ecosystems of ecosystems. As an analytical construct, this leads to a problem of stakeholder inclusion: which level of analysis to use and where to draw the borders for an ecosystem? The problem of inclusion is not unique to ecosystems, but is a classical problem in social theory that has been discussed extensively in prior literature [22].

Fourth, characteristic of ecosystems is that they are not stable, but are constantly evolving. Continuous adaptations in the relationships between ecosystem stakeholders across the micro, meso and macro-levels make ecosystems dynamic. The systemic properties of an ecosystem means that changes in one of the ecosystems entities will trigger responses and changes in other entities. As a consequence, "ecosystems are essentially defined by the active shaping of relationships between their members" [33:2].

Previous research has observed that ecosystems transform rapidly along with technological innovations that alter conditions for the business landscape $[14,45,46]$. New technology generations are fundamentally reshaping the traditional logic, as business processes become intertwined with surrounding technology ecosystems [4]. This is also true in the software ecosystems.

We conceptualize a software ecosystem as one that exists in a fusion relation [13] to technologies, in which the technologies form part of a technology ecosystem. Adomavicius et al. [1:201] define a technology ecosystem as "system of interrelated technologies that influence each other's evolution and development." The definition is based on the view of evolving components of complex technologies as being mutually interdependent 
$[15,17]$. Consequently, from the consumer side, the selection of a specific technology is associated with a decision to join a particular business network that operates in a specific technological regime with interoperable technologies [35].

\section{Methodology}

We conduct a revelatory case study [44]. A revelatory case is a case that gives the researchers the unique opportunity to investigate the phenomenon of interests (ibid). The case company for our study, ERP $\mathrm{Co}^{1}$, is a leading vendor of various enterprise systems in the SMEs (small and medium enterprises) market.

For the purpose of this paper, we investigate the (still on-going) process of which ERP Co made the shift towards a SaaS-based business model. The company was chosen because of its revelatory nature in 1) understanding the business transition associated with technological changes; 2) revealing the change dynamics across the whole ecosystem [44].

\subsection{Data collection and analysis}

Primary source of data has been interviews with six key informants from the top management, including innovation managers and representatives for the new SaaS platform. An interview guide was prepared with short introduction to the approach and setting for the interview and questions that would act as guide through the interview. The questions were structured to provide input on various aspects of ERP Co's transition towards the new business model. For example: "Which changes will SaaS introduce for ERP Co's distribution channel partners?" This was designed to collect an understanding of the interviewees' view on the impacts of SaaS on the whole business network.

With the primary data in place the analysis of primary data could be initiated. Participant opinions were reviewed and analyzed to verify the sufficiency of the collected data and the suitability for the later research impact on the business ecosystem during transition at ERP Co.

\subsection{Case background}

ERP Co is a leading vendor of enterprise resource planning (ERP), customer relationship management (CRM), and human resource management (HRM) systems in the European market for small and medium sized enterprises (SME).

\footnotetext{
${ }^{1}$ A pseudo name was used to ensure anonymity of the company.
}

ERP Co was founded in the mid-1990s as provider of marine software. The current CEO joined in 1997 when the company was at the brink of bankruptcy. The first strategic choice made as the new CEO was to switch the focus of ERP Co toward providing "need-to-have" software rather than "niceto-have" software. For example, software that supports external accounting, tax reporting, and payroll became the new focus of ERP Co. In 2000, ERP Co sold the marine division for around 80 million Euro in cash that was then used to acquire the top competing software companies as well as accounting offices.

From 2001 to 2006, the company continued its growth through acquiring companies that have technical talent and low profit margin. In 2006, ERP Co has grown to a company with 3000 employees.

In 2006, a financial buyer acquired ERP CO for 4.3 billion. The acquisition resulted in a couple of changes: 1) the management team stayed the same but have to deliver on a set of KPI (key performance indicator) every year; 2) top 100 managers obtained stock from the company, which created the sense of ownership. In 2010 and 2014, ERP Co experienced another two rounds of acquisition by financial buyers, for 1.3 billion Euros and 2.5 billion Euros respectively. In 2014, ERP Co has 6,000 employees, and achieved 800 million NOK in revenue.

\section{Findings}

\subsection{ERP CO before transition to cloud}

ERP Co can be divided into three segments by three major products/services it offers. The first division is called software SMB (small-medium business) which provides ERP, payment, HR, CRM systems to small and medium size customers. The customer segments can be further divided into three categories. First, we have micro size business, which out of box standardized products are directly sold to. ERP Co provides product training online and a helpdesk for customer services, but nothing more (e.g., on-site training or consulting services). The second segment is small and medium size business with less than 1000 employees, where ERP Co works closely with their partners, all what they refer to as value added resellers. Here, all the sales go through ERP Co partners, which could be platinum partners that only work for ERP Co and build their services around ERP Co products, or gold partners that mainly work with ERP Co but also engage with other businesses, or smaller partners and resellers that represent ERP Co or their products in micro markets 
and local markets around the country. Customers in this segment can obtain tailor made system based on standard ERP Co product through ERP Co partners. The third customer segment is bigger $\mathbf{S M B}$, where intensive consulting is required. This segment is currently not the focus of ERP Co.

The second division of ERP Co provides BPO (business process outsourcing) services that mainly involve accounting, payroll, and advising related products. The last division of ERP Co deals with government and large retail accounts, which only started several years ago. In this study, we will only focus on the software SMB division of ERP Co.

Figure 1 presents the ecosystem of ERP Co software. The main stakeholders here include the three groups of customers and three types of partners.

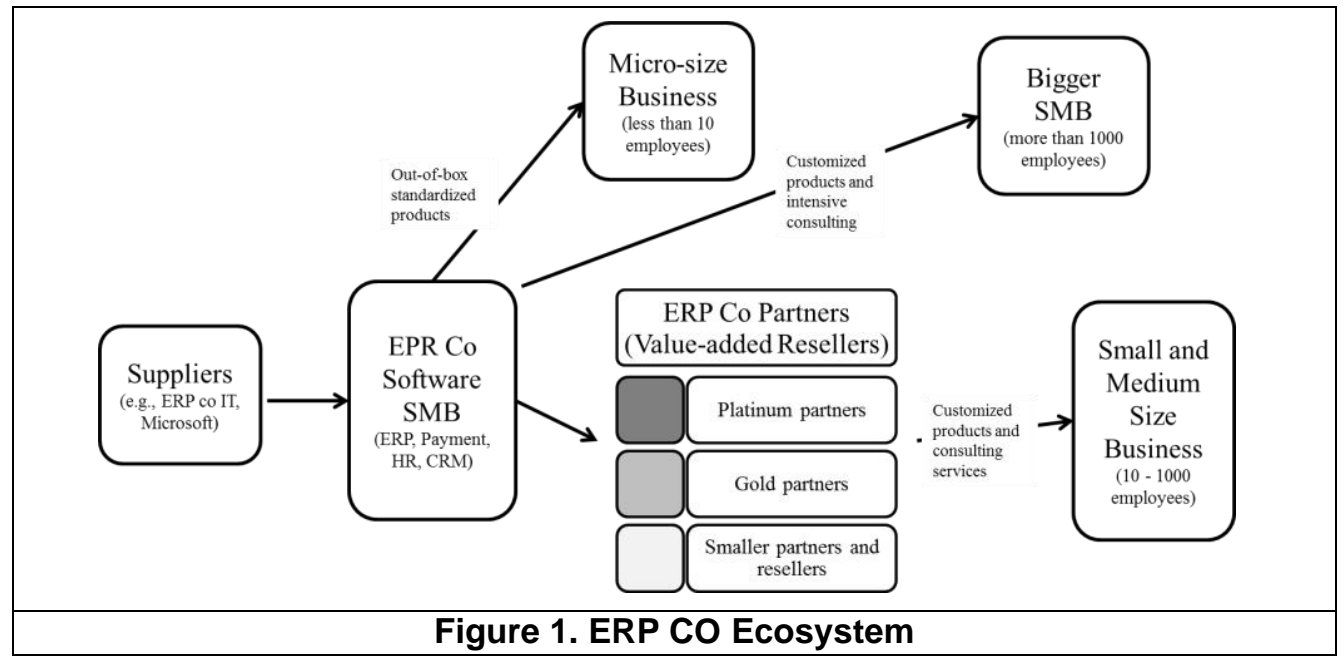

VAR is a critical part of ERP Co's business ecosystem, as they are in direct charge of selling to the largest customer segment, the small and medium size businesses. In this segment, VAR are either resellers, or partners who develop customized products and services around ERP Co products for certain industries, and up to $50 \%$ of the initial ERP Co license fees do go to VAR. Except for the platinum partners who work solely with ERP Co, other partners maintain a co-petition relationship with ERP Co as they also engage with other products/services of similar nature. However, it is safe to say that such partnership between ERP Co the VAR is a crucial component of ERP Co's business model and the subsequent ecosystem built around ERP Co software. On one hand, the reliance on VAR to develop tailored systems and services to customers from various industries with diversified needs implies that ERP Co can only focus on the development and maintenance of the standardized product; on the other hand, the representation of ERP Co to micro and local markets through partners grants them the opportunities to penetrate the market in an efficient and effective way. Similarly, these partners/resellers heavily depend on ERP Co, some more so than others, for the survival/thrive of their own business.

On the supplier side, their major providers include ERP Co IT (a company owned by ERP Co) and Microsoft. It is worth noting that since ERP Co develops most of its products in-house, the upstream distribution channel is rather simple, and hence will not be the focus of this study.

In summary, ERP Co operates within an ecosystem where close dependencies among stakeholders exist. Therefore, it can be speculated that changes among major players will likely to cause certain ripple effects that result in evolution (or even collapse) of the ecosystem [18].

\subsection{ERP Co's transition to cloud}

\subsubsection{From 2011 to 2013}

Though ERP Co's SaaS journey has started since early 2000 by providing some simple applications through the web, the official transition towards a cloud-based business model was initiated around 2011, when ERP Co acquired an US-based SaaS provider. The decision for the transition was on the one hand partially triggered by the macro environment with increasing market demands and technological maturity, and on the other hand pressured by the investor at the time as transition to cloud is likely to increase the value of the company.

Indeed, the pressure from the investor in terms of the speed of the transition has caused certain initial struggles for ERP Co. Upon the acquisition of the SaaS vendor as mentioned above, ERP Co adjusted 
their research and development resources to $80 \%$ in SaaS and $20 \%$ in on-premise, as an attempt to facilitate the development of the SaaS products and hence the transition to cloud. Such strategic move has directly and indirectly caused a number of challenges. To begin with, on the product side, ERP Co has realized the difficulties in develop a sound SaaS product. Though they have acquired the SaaS software from US, they under-estimated the efforts in adjusting the product to local rules and contexts (e.g., certain financial procedures are very different in the US than in the Nordic countries). Meanwhile, due to the decrease in $R \& D$ allocation for on-premise software, the quality of the product became a problem subsequently. Therefore, ERP Co was put in a situation where SaaS products took longer than expected while at the same time no new products can be offered as alternative. Further, the KPIs (key performance indicator) at the same were based on the sales of on-premise products, and rendered as a conflict to the transition to cloud.

Such strategic transition to cloud has already created rippling effects in the ecosystem. First, the customers and partners show uncertainties towards cloud and SaaS products, as we have seen in the history of innovation adoption. On the customer side, ERP Co admitted the underestimation of the "stickiness" of on-premise products. The common reaction from the customers towards ERP Co's strategic transition to cloud-based products is concern: "Are you not going to support the old product any more?" In other words, how to maintain the trust from the customers, and bring them on board has become a challenge. Additionally, triggered by the quality drop of the on-premise product, as mentioned above, customers feared that support and maintenance of existing on-premise systems that they have already purchased will not be provided by ERP Co as they saw the company making such a speedy shift towards cloud, causing further panic among loyal supporters of the company.

On the VAR side, the problem is even bigger. First, partners will not sell unfinished products, and therefore, they will not be bought in unless the SaaS product from ERP Co is fully developed. As mentioned earlier, many of ERP Co's partners are indeed in a co-opetition relationship with the company, meaning that they will sell other products from ERP Co's competitors if they don't see the value of the new SaaS product.

Furthermore, the partners fear the termination of the partnership given that the nature of SaaS model means implies disintermediation to a certain degree. As put by one of the managers, a key question that has risen in every partner's mind is "...given there is nothing to resell, nothing to technically install and no opportunity to provide any kind of logistics, what can a successful SaaS partner contribute?" In this sense, whether and how to support partners' business model that is on the edge of erupting in the new environment is also of great concern for ERP Co to maintain their ecosystem.

Finally, as discussed at the beginning of the paper, the pay-as-you-go or subscription-based pricing model associated with cloud and SaaS presents a challenge to both ERP Co the vendor and its partners in managing their revenue streams.

\subsubsection{From 2014 on}

These challenges and problems persisted from 2011 to 2013, as ERP Co was struggling to find out a way to make the transition go sound and smooth. A solution arrived in 2014 when the management recognized the issues in rushing towards cloud and making transitions overnight, and hence fell back on the on-premise model and started to take things slowly.

The resulting strategy is to pursue the so-called hybrid model where both ends (SaaS-based and onpremise products) were handled in equal terms. The distribution of $\mathrm{R} \& \mathrm{D}$ resources was adjusted to $50 \%$ each for SaaS products and on-premise products. The direct effect of such adjustment is that the quality of on-premise products has been increased and back to the usual level. The hybrid strategy led to so-called "controlled transition" towards cloud, which heavily involves all the stakeholders in the ecosystem. More specifically, on the customer side, ERP Co conducted a round of pilot implementation by selling $50 \mathrm{SaaS}$ product to approved customers who met the criteria for SaaS implementation (in terms of organizational readiness for SaaS). In other words, sales of the SaaS product was done in a controlled environment for the purpose of minimizing negative effects in case of functionality/usability related issues. Doing so, ERP Co has gained valuable feedback from the customers for further development and improvement of the SaaS product, while at the same time easing the customers into the transition process. As for other customers who have on-premise core systems, ERP Co will on the one hand keep maintaining and supporting the on-premise product (reflected in the re-adjustment of R\&D investment), and one the other hand selling SaaS-based add-ons. The goal as of 2014 for ERP Co is to start selling SaaS products to all the new customers in 2015, especially for the segment of micro business who mostly rely on standardized outof-the-box software. 
As discussed earlier, ERP Co's partner model faces serious challenges triggered by the transition to cloud, as the role and business model for the partner have to be redefined in the new context where no physical software is sold and installed. When asked about the possibility of maintaining the VAR model in the SaaS environment, the response from ERP Co management is positive: the strategy is to go "hand in hand" with partners to make the transition. The envisioned change regards three main areas. First, the revenue model associated with SaaS means that the upfront perpetual licenses fees would be replaced by long-term flowing of subscription fee. Hence, ERP Co is looking to compensate some of the partners (especially the ones that solely rely on ERP Co) for the initial years for the sales of SaaS products (higher percentages of subscription fees will go to the partners, for instance). Second, the correspondently, the sales model of partners is also subject to change due to the change in revenue model. Now partners should look for expansion of sales channels to increase quantity of sales to compensate for loss in short-term cash flow. Third, as the requirements for technical configuration for SaaS products are lower, compared to the on-premise context, the services offered by partners should be shifted away from technical consulting towards business process consulting.

\section{Discussion}

The ERP Co case we have presented above demonstrates a landscape shift of an existing, wellestablished business ecosystem, triggered by technological innovations [14]. In the current analysis of ERP Co's transition to cloud, we have focused mostly on the downstream distribution side of the ecosystem, i.e. its distributors, as the company mostly develops their products in-house. Though the shift towards SaaS products does imply changes in the supplier market (e.g., type of components that need to be purchased and the development tools and the skills need to develop a cloud based service), the nature of the exchange relationship has not been influenced as much as the relationship with downstream customers and partners.

\subsection{Evolution of the ecosystem triggered by technological changes}

The transition process ERP Co has gone through so far, including the initial struggles, showcases the extend of which technological innovations can bring to an industrial value chain and business communities $[1,29]$. The initial ecosystem on which the success of
ERP Co was built upon involves multiple stakeholders and correspondent sets of relationships that are based on the specified product the company offers and the business model it operates in. For instance, ERP Co only offers standardized product, which is sold either in an out-of-box fashion to micro business, or through partners who make customizations for specific customer or market.

It can be argued that the VAR model is at the center of the old ecosystem: mediating the exchange relationship between ERP Co and its largest customer segment. The division of partners into three categories shows different types of stakeholder relationships and corresponding collaboration modes [18]. For instance, the platinum partners collaborate with ERP Co to achieve "synergistic integration," in which new products and services are developed by the partners based on the ERP Co system, and both sides reinforce each other and achieve value cocreation, whereas gold partners and smaller partners might mainly engage in "addition" or "exchange" type of collaboration with ERP Co, where they simply redistribute ERP Co products or provide customization and implementation services [12,31].

As discussed earlier in the paper, though often marked as a technological innovation, cloud computing represents a new way of which IT resources are delivered and consumed [2]. Hence, for ERP Co, the transition means more than just the development of the new SaaS-based offering. The fact that customers can access their enterprise systems through a web browser means that the process involved in delivering and installing a physical product on customers' local computers and device has been eliminated and bypassed. In other words, the nature of the product and related business process (sales process more specifically), upon which the old ecosystem was built on, has changed. ERP Co's efforts in managing the transition can therefore be interpret as a process of redefining the stakeholder relationships under the new circumstances [33]. Consequently, the ERP Co's partners might have to reinvent themselves, and provide another type of value to the end users.

The categorization of three types of partners, associated with their different modes of collaborating with ERP Co, represents the concept of the observable differences within ecosystem. In other words, we find that some stakeholders are more visible in the network and have stronger relationships with each other, in particular the platinum VAR. The idea of labeling some partners as platinum partners is signal to the ecosystem that some are more important than others. Our case shows that these observation differences need to be taken into considerations when 
redefining the stakeholder relationships. ERP Co indeed supported its partners' adaptations in different ways: while all the partners would obtain a certain level of compensation in selling SaaS products, it is the platinum partners that ERP Co worked "hand-inhand" with, to develop new services that can be built upon the SaaS product and achieve synergistic integration. These new services, as put earlier by one of ERP Co's managers, are shifting toward business process consulting and away from technical consulting, due to the nature of SaaS products.

Besides the long relationship between ERP Co and the partners, the partners and ERP Co are in coopetition (especially for the gold partners and smaller partners), since some of them are also selling competing ERP software. This is also true for the main vendor - Microsoft - who sells competing software on global scale. This makes the relationships in the ecosystem sensitive and requires careful management. Thus the technological evolution enables new forms of competition, where firms have to collaborate with its competitors and clients.

To sum up, we find support of that ecosystem - as in nature - is not a stable entity. They evolve and in our case due to technological evolution and firms' ability to adopt and change to these innovations. Thus to survive in ecosystem requires adaptations both upwards and downwards at micro, meso and macrolevels. ERP Co's transition is a consequence of that "ecosystems are essentially defined by the active shaping of relationships between their members" [33:2]. The re-shaping of the exchange relationships among the stakeholders of ERP Co's ecosystem is a direct result of technical innovations replacing the product that is at the center of the exchange relationships.

\subsection{Managerial implications for transition to cloud}

The analysis of the case of ERP Co has also generated important managerial implications for IT vendors who are going through similar transition process to cloud. Here we have listed the following challenges/ critical aspects in successfully managing such change in business.

First and foremost, as discussed thoroughly above, transition to cloud for traditional IT vendors represents more than just adoption of new technology and development of new product. Rather, transition to cloud needs to be managed as a change of the whole business model and business network. The point we are trying to make here is that the management needs to fully prepare the company for the scale and magnitude of the change brought by the transition to cloud. As put by one of the directors at ERP Co: "I believe one of the most critical success factors are what you can call organizational preparedness, since this goes so deep in our ability to understand and implement decisions throughout the value chain." In other words, the inter-dependencies among ecosystem stakeholders need to be taken full consideration of by the vendor, who should manage the relationship with these stakeholders accordingly. For instance, ERP Co's strategy towards the partners is "hand-in-hand" transition and joint reconfiguration of the business process. Constraining the scale of change within the organization might cause the collapse of the ecosystem, which in turn threatens the survival of the business itself.

Second, hybrid model not only applies for cloud adopters, but also for vendors who manage a transition from on-premise products to cloud-based products. As seen in the case, the initial struggles from ERP Co were caused by the speed of which they tried to pursue the transition, which resulted in panics both within the organization, and across the value chain to the customers and partners. The later adjustment to a "hybrid strategy" led to smoother transition process, as keeping and maintaining onpremise products 1) buys more time for developing new SaaS products without sacrificing existing marketing share due to lack of finished products to sell, and 2) provides a chance for both the customers and partners to gradually adapt to the change.

Third, transition to cloud needs to be matched with and supported by changes in other parts of the business. We have observed changed across many business areas of ERP Co, and also problems triggered by lack of changes in certain areas initially. Operating under a cloud-based business model demands changes in areas such as revenue management (up-front bulk payment versus continuous cash flow), sales channel (coordination with partners), customer support and customer relationship management, and so on. Hence, the management needs to map out all the change areas and make adjustments accordingly. For instance, in the ERP Co case, the original KPIs that directly correlate with sales of on-premise products did not align with the shift towards cloud, and therefore resulted in lack of motivation from within the organization.

\subsection{Limitations and future research}

The most important limitation of the research in this article is that it only investigates one part of the ecosystem. Subsequent research should therefore 
expand to include the relationship between distributors and end user firms and investigate the three-way dynamics (among vendors, distributors, and end customers), which is a future step for our study. Additionally, our work points out that the rising of cloud computing has important implications for the business model and the underlying profitgenerating mechanisms of the firm. Thus future studies on ecosystem transition should also dwell into how profit generations are changing. Furthermore, we encourage researchers to include more cases for both the vendor perspective and the B2B context when engaging in cloud computing related research.

\section{Conclusion}

In this paper, we have explored the changes of B2B software partnership due to technological innovation from the lens of ecosystem. Based on a case of ERP Co, a leading enterprise software vendor in the SME market, we have uncovered the process of which transition to cloud has occurred to date, and its rippling effects towards the whole business ecosystem. Among the transition, we find new types of relationships emerging among the stakeholders, increasing co-opetition and the visibility of some partners, and that the ecosystem is not stable and evolves with technological changes. Based on these insights, we offer managerial implications for IT vendors in making the transition to the cloud.

\section{References}

[1] Adomavicius, G., Bockstedt, J.C., Gupta, A., and Kauffman, R.J. Technology roles and paths of influence in an ecosystem model of technology evolution. Information Technology and Management 8, 2 (2007), 185-202.

[2] Armbrust, M., Stoica, I., Zaharia, M., et al. A View of Cloud Computing. Communications of the ACM 53, 4 (2010), 50.

[3] Bardhan, I.R., Demirkan, H., Kannan, P.K., and Kauffman, R.J. Special Issue: Information Systems in Services. Journal of Management Information Systems 26, 4 (2010), 5-12.

[4] Basole, R.C. Visualization of interfirm relations in a converging mobile ecosystem. Journal of Information Technology 24, 2 (2009), 144-159.

[5] Benlian, A. and Hess, T. Comparing the relative importance of evaluation criteria in proprietary and opensource enterprise application software selection - a conjoint study of ERP and Office systems. Information Systems Journal 21, 6 (2011), 503-525.
[6] Benlian, D.A., Hess, P.D.T., and Buxmann, P.D.P. Drivers of SaaS-Adoption - An Empirical Study of Different Application Types. Business \& Information Systems Engineering 1, 5 (2009), 357-369.

[7] Brandenburger, A.M. and Nalebuff, B.J. Co-Opetition. Crown Publishing Group, 2011.

[8] Columbus, L. Demystifying Cloud Vendors. Forbes, 2013.

http://www.forbes.com/sites/louiscolumbus/2013/02/20/de mystifying-cloud-vendors/.

[9] Columbus, L. Roundup Of Cloud Computing Forecasts And Market Estimates, 2015. Forbes, 2015.

http://www.forbes.com/sites/louiscolumbus/2015/01/24/rou ndup-of-cloud-computing-forecasts-and-market-estimates$2015 /$.

[10] Creeger, M. CTO Roundtable: Cloud Computing. Quеие 7, 5 (2009), 1:1-1:2.

[11] Cusumano, M. Cloud computing and SaaS as new computing platforms. Commun. ACM 53, 4 (2010), 27-29.

[12] Das, T.K. and Teng, B.-S. A Resource-Based Theory of Strategic Alliances. Journal of Management 26, 1 (2000), $31-61$.

[13] El Sawy, O.A. The IS Core IX: The 3 Faces of IS identity: connection, immersion, and fusion. Communications of the Association for Information Systems 12, 1 (2003), 39.

[14] Evans, D.S., Hagiu, A., and Schmalensee, R. Invisible engines: how software platforms drive innovation and transform industries. MIT Press Cambridge, MA, 2006.

[15] Henderson, R.M. and Clark, K.B. Architectural Innovation: The Reconfiguration of Existing Product Technologies and the Failure of Established Firms. Administrative Science Quarterly 35, 1 (1990), 9-30.

[16] Hsu, P.-F., Ray, S., and Li-Hsieh, Y.-Y. Examining cloud computing adoption intention, pricing mechanism, and deployment model. International Journal of Information Management 34, 4 (2014), 474-488.

[17] Iansiti, M. and Khanna, T. Technological Evolution, System Architecture and the Obsolescence of Firm Capabilities. Industrial and Corporate Change 4, 2 (1995), 333-361.

[18] Iansiti, M. and Levien, R. The Keystone Advantage: What the New Dynamics of Business Ecosystems Mean for Strategy, Innovation, and Sustainability. Harvard Business Press, 2004. 
[19] Iyer, B. and Henderson, J. Preparing for the future: understanding the seven capabilities of cloud computing. MIS Quarterly Executive 9, 2 (2010), 117-131.

[20] Kasznik, E. 'Churn is king': how SaaS business models affect software company valuations. Intellectural Asset Management, 2014. http://www.iammagazine.com/industryreports/Detail.aspx?g=fc2623953a71-4a6e-8828-6c6c6b7e429a.

[21] Luftman, J. and Zadeh, H.S. Key information technology and management issues 2010-11: an international study. Journal of Information Technology 26, 3 (2011), 193-204.

[22] March, J.G. and Simon, H.A. Organizations. Wiley, Oxford, England, 1958.

[23] Marston, S., Li, Z., Bandyopadhyay, S., Zhang, J., and Ghalsasi, A. Cloud Computing - the Business Perspective. Decision Support Systems 51, 1 (2011), 176-189.

[24] Moore, J.F. Predators and prey: a new ecology of competition. Harvard business review 71, 3 (1993), 75-83.

[25] Nikolov, G.I. Cloud Computing and Government: Background, Benefits, Risks. Nova Science Publishers, Inc., Commack, NY, USA, 2011.

[26] Oliveira, T., Thomas, M., and Espadanal, M. Assessing the determinants of cloud computing adoption: An analysis of the manufacturing and services sectors. Information \& Management 51, 5 (2014), 497-510.

[27] Owens, D. Securing Elasticity in the Cloud. Commun. ACM 53, 6 (2010), 46-51.

[28] Parameswaran, S., Venkatesan, S., Gupta, M., Sharman, R., and Rao, H.R. Impact of Cloud Computing Announcements on Firm Valuation. AMCIS 2011 Proceedings - All Submissions, (2011).

[29] Rosenkopf, L. and Tushman, M.L. The Coevolution of Community Networks and Technology: Lessons from the Flight Simulation Industry. Industrial and Corporate Change 7, 2 (1998), 311-46.

[30] Ryan, M.D. Cloud Computing Privacy Concerns on Our Doorstep. Communications of the ACM 54, 1 (2011).

[31] Sarker, S., Sarker, S., Sahaym, A., and BjørnAndersen, N. Exploring Value Cocreation in Relationships Between an ERP Vendor and its Partners: A Revelatory Case Study. MIS Q. 36, 1 (2012), 317-338.

[32] Schneider, S. and Sunyaev, A. Determinant factors of cloud-sourcing decisions: reflecting on the IT outsourcing literature in the era of cloud computing. Journal of Information Technology, (2014).
[33] Selander, L., Henfridsson, O., and Svahn, F. Transforming Ecosystem Relationships in Digital Innovation. ICIS, (2010), 138.

[34] Selander, L., Henfridsson, O., and Svahn, F. Capability search and redeem across digital ecosystems. Journal of Information Technology 28, 3 (2013), 183-197.

[35] Shane, S. Technology Regimes and New Firm Formation. Management Science 47, 9 (2001), 1173-1190.

[36] Smith, D.M. Cloud Computing Changes the Vendor Landscape. 2012.

http://www.gartner.com/it/content/2154700/2154716/octob er_3_cloud_computing_dsmith.pdf?userId=74657470.

[37] Son, I. and Lee, D. Assessing A New IT Service Model, Cloud Computing. PACIS 2011 Proceedings, (2011).

[38] Tansley, A.G. The Use and Abuse of Vegetational Concepts and Terms. Ecology 16, 3 (1935), 284-307.

[39] Vargo, S.L. and Lusch, R.F. Evolving to a New Dominant Logic for Marketing. The Journal of Marketing 68, 1 (2004), 1-17.

[40] Venters, W. and Whitley, E.A. A Critical Review of Cloud Computing: Researching Desires and Realities. Journal of Information Technology 27, 3 (2012), 179-197.

[41] Walley, K. Coopetition: An Introduction to the Subject and an Agenda for Research. International Studies of Management \& Organization 37, 2 (2007), 11-31.

[42] Willcocks, P.L.P., Venters, W., and Whitley, D.E.A. Moving to the Cloud Corporation: How to face the challenges and harness the potential of cloud computing. Palgrave Macmillan, Houndmills, Basingstoke, Hampshire, 2013.

[43] Winkler, T.J. and Brown, C.V. Horizontal Allocation of Decision Rights for On-Premise Applications and Software-as-a-Service. Journal of Management Information Systems 30, 3 (2013), 13-48.

[44] Yin, R.K. Case Study Research, Design and Methods. Sage Publications, Newbury Park, CA, 2003.

[45] Yoo, Y., Lyytinen, K., and Boland, R.J. Innovation in the digital era: Digitization and four classes of innovation networks. Hawaii International Conference on System Sciences, (2008).

[46] Zammuto, R.F., Griffith, T.L., Majchrzak, A., Dougherty, D.J., and Faraj, S. Information Technology and the Changing Fabric of Organization. Organization Science 18, 5 (2007), 749-762. 